\title{
Cognitive science: The art and its implications
}

\author{
Boris M. Velichkovsky \\ National Research Center "Kurchatov Institute", Moscow, Russia \\ M.V. Lomonosov Moscow State University, Moscow, Russia \\ Russian State University for the Humanities, Moscow, Russia \\ Moscow Institute for Physics and Technology, Moscow, Russia \\ Technische Universität Dresden, Germany
}

Contemporary cognitive science is the latest version of the century-long quest for a better understanding of the human mind and brain. Various disciplines have brought together empirical methods and theoretical models from their fields of study to further this effort. This multidisciplinary convergence widely known today by the acronym $\operatorname{NBIC}(S)^{1}$ is the general context for the present endeavor.

Why is a journal with "Psychology" in its title concerned with this development? In fact, those were the founding fathers of psychology, physicist Hermann von Helmholtz and physician Wilhelm Wundt, who demonstrated the conviction that psychology as science can only be one of interdisciplinary kind. In the 20th Century, Jean Piaget gave psychology a central place among the sciences, because, in his view, only psychology studies the conscious mind that makes science and critical thinking possible. But he insisted that he was not a psychologist, explaining that he studied "epistemic" rather than "psychological" issues. In a similar vein, some psychologists would say today that they rather belong to a multidisciplinary cognitive community whereby adjective "cognitive" replaces here what Piaget called "epistemic" half a century ago.

This is the case of authors participating with their papers in this special issue of Psychology in Russia. Many of them are not psychologists by training, and they do their professional work in fields as varied as mathematical physics, neuroimaging, molecular biology, and the pragmatics of communication. Nevertheless, their con-

1 As in the name of the Complex of NBICS-technologies at the National Research Center "Kurchatov Institute", where the capitalized letters stay for Nanotechnology, Biotechnology, Information technology, Cognitive, and Social sciences. 
tributions to the journal's topic, and, in my opinion, to the future of psychology as a science, are preeminent. Overall, in order to solve our century-long problems, we have to look to a diversity of approaches, and combine them in a convergent way. This is the first implication to be drawn from contemporary cognitive science.

The second implication is that emphasis must be placed on application and technologies. It is no accident that NBICS studies deal with technologies, not endless verbal or even experimental exercises in an ivory tower. Cognitive psychology, with its bias for academic sterility, was for too long deprived of almost any practical significance. But the proof of the "cognitive pudding" is in the practical "eating." The emphasis on applications does not mean the degradation of basic research. There are numerous links between basic and applied cognitive studies, which once again show that there is nothing more practical than a good theory. It can easily be demonstrated, from the research being done on perception, attention, memory, and communication, that every scientifically established fact about the organization of human cognition has important practical consequences. If not, then we simply were yet unable to recognize how the results could be applied in the major domains of praxis - engineering, medicine, and education.

The environments in which we carry out our everyday activities are complex, dynamic, sometimes dangerous, and demanding demanding a lot of social of social skills - even wisdom. From a purely quantitative point of view, they are producing streams of data that vastly exceed the known limits of human cognitive capacities. However, in striking contrast to existing "intelligent" technical systems, we, as a rule, easily understand and intelligently act in everyday situations. How do people use their perplexed brain machinery and notoriously limited cognitive resources to manage ever increasing environmental demands? The topics dealt with in this special issue have a more or less direct relationship to the answer to this question. We suspect that the general answer is called "consciousness," with its variety of derived abilities such as understanding, intellect (at least, the fluent version of it), and higher-order emotions and feelings.

Heinz Heckhausen once remarked that scientific psychology first banished the soul from its lexicon, and then lost consciousness and the ability to reason. With the rise of cognitive psychology in the second half of the $20^{\text {th }}$ Century, reasoning and other cognitive functions have been returned from their behaviorist exile. Today, we see an unprecedented expansion of cognitive and neurocognitive studies of consciousness. ${ }^{1}$ Hope is stronger than ever that this is the time to tackle big research questions. Perhaps, this is an illusion, like consciousness itself, but the emphasis on consciousness and volition is clearly seen in the collection of articles in this issue. Among the articles, there are research papers directly dedicated to phenomenology and to the brain's mechanisms of consciousness and, symptomatically, to practical applications in such domains of new and emerging technologies as virtual reality ("feeling of presence") and eye-brain-computer interfaces ("intentions," "sense of agency").

The appeal to ask questions that could not be answered for decades and centuries is of limited value unless we have powerful research tools for reaching the

1 By way of analogy, one can ask about the possible return of the soul in scientific discourse. This is perfectly conceivable but not before both trends - cognitive and affective research - are united in something like cognitive-affective science (see, e.g., Panksepp, 1998). 
answers. Here we are again at a crossroads, with NBICS-convergence being the basis for the development of such research methods: from non-invasive brain imaging and eye-tracking instruments with their specialized mathematical tools, to the methods of genomic, epigenetic and metabolomic studies revealing underlying molecular mechanisms.

This convergence of methodologies seems to lead to a convergence in results. To be more specific, I will address several implications of the recent discovery which demonstrated a profound asymmetry in effective (cause-and-effect) connections between the left and right hippocampi within the human default mode network, or DMN (Ushakov et al., 2016). The latter is a set of functionally and structurally connected brain areas that are activated at rest and deactivated by external stimulation. The lateralization of the neocortex has been a well-established fact since the famous observation by Paul Broca that the left hemisphere plays a crucial role in speech production ("Nous parlons avec l'hémisphère gauche"). However, our finding is a novum because, for the first time, it refers to an asymmetry in the relationship between subdivisions of the limbic system, i.e. relatively deep structures of the paleocortex.

Moreover, the pattern of causal relationships, which is characteristic for the right hippocampus, seems to be of particular importance for self-referential cognition and higher-order forms of emotional life. The key feature of this pattern is a holistic representation of one's egocentric surroundings. With the centrality of Ego/Self in such a representation, it can provide an easy-to-find gateway into much of what we used to call "subjective experience." The access to the gateway seems to be open both for prefrontal cortices and for tertiary associative structures around right temporoparietal junction in the posterior part of the human brain (Velichkovsky et al., 2017).

Several works in this collection of papers seem to support this hypothesis in a number of research domains, at least indirectly. All of them highlight the rightward lateralization of brain mechanisms for self-referential processing. Besides the phenomenology of everyday problem-solving and neuropsychological testing in patients with unilateral brain lesions, this research includes neuroimaging studies of reading, remembering, and cognitive involvement in motor behavior. Last but not least, a pronounced difference in the gene expression within the left and right parts of the human frontopolar cortex is reported (Dolina et al., 2017). Here again, a strong right-sided predominance in the differential expression of protein-coding genes was established. ${ }^{1}$ Many of these differentially expressed genes are known for their role in hippocampal formation and, if considered in a clinical context, for their relationship to schizophrenia.

Although basic mechanisms of language perception and production, localized primarily in the left hemisphere, have been traditionally considered as the differentia specifica of Homo sapiens, parts of the right hemisphere have a larger volume and demonstrate more rapid growth, both in anthropogenesis and in early ontogenesis. This fact can be related to the Yakovlevian Torque phenomenon, in which frontal structures anterior to the right Sylvian fissure are "torqued forward" relative to their counterparts on the left. This phenomenon has been supported

1 The pattern of asymmetry in up-expression is less apparent, missing, or even completely reversed toward leftward asymmetry if we are considering short non-coding molecules, i.e. microRNA and its precursors (Nedoluzhko et al., in preparation). 
by fragmented paleo-neurological findings dating back nearly one million years (Hrvoj-Mihic, Bienvenu, Stefanacci, Muotri, \& Semendeferi, 2013). It looks as if we are unique among species not for our language ability but rather for our elaborated mechanisms of controlling what we are saying and doing. Thus, the upper-level mechanisms may precede and definitively influence, in a top-down manner, the initial phases in evolution of human language (Deacon, 1996).

Instead of going into details on this multilevel brain-and-mind architecture (see, e.g., Velichkovsky, 1999, 2002), I will only stress the coherence among the empirical findings. The right hemisphere was for decades considered as subordinate and undeveloped, at best specialized in spatial information processing. However, the new findings show that it is crucially important for self-referential cognition, theory of mind, and higher forms of emotional comprehension. An unexpected result also is that not only prefrontal mechanisms are involved, but posterior structures near the temporoparietal junction are as well. Within the DMN circuitry, a common target of these widely separated cortical mechanisms are limbic structures in the depths of the right hemisphere. Our group's discovery of the asymmetry in hippocampal causal connections now starts to be completed by the data on the analogous uniqueness in the functional connectivity of the right amygdala (Kerestes, Chase, Phillips, Ladouceur, \& Eickhoff, 2017).

William James in his Principles of Psychology (1890) used the notion "primary memory," which was for him the equivalent of the field of consciousness - the scope of the content which we are aware of at the present moment, in the absence of physical stimulation. A bit later, Henry Bergson (1907/2006) noted that to study authentic consciousness, one has to exclude external stimulation. This reminds us of two contemporary concepts. The first is the DMN, a brain circuitry which is activated in the resting state, and is, according to the emerging knowledge, related to introspection and other aspects of consciousness. The second concept is working memory. It is a modern counterpart to James's primary memory and one of the central concepts in cognitive science because of its relevance to clinical data, intelligence measurement, and academic achievements. Recent neuroimaging studies of the DMN bring together both concepts by showing that activity around the right temporoparietal junction is the best predictor of working memory scores (Markett et al., 2017). This region in turn is the main input pathway to the right hippocampus within the DMN connectome (see Ushakov et al., 2016, Velichkovsky et al., 2017).

The ability to retain, manipulate, and invent information in the absence of external stimulation is a key prerequisite for mental life and for goal-directed interaction with the environment. However, how this ability is implemented in the brain wetware remains one of the great mysteries of psychology and neuroscience. The current collection of research papers gives us some hope that we are approaching a solution to this century-long problem.

\section{Acknowledgments}

This introduction and the majority of papers in this special issue of Psychology in Russia have been supported by the Russian Science Foundation (grants RScF 14-28-00234 and 17-78-30029 aimed at investigation of self-referential cogni- 
tion and semantic representations in the human brain, respectively). Permanent support on the part of the journal editorial team is appreciated. Special thanks are due to T.O. Archakova, A.N. Veraksa, and Yu.P. Zinchenko.

\section{References}

Deacon, T.W. (1996). Prefrontal cortex and symbol learning: Why a brain capable of language evolved only once. In B. M. Velichkovsky \& D. M. Rumbaugh (Eds), Communicating meaning: The evolution and development of language (pp. 103-138). Mahwah NJ: LEA Publishers. doi: 10.1111/j.1467-9744.1996.tb00954.x

Dolina, I.A., Efimova, O.I., Kildyushov, E.M., Sokolov, A.S., Khaitovich, P.E., Nedoluzhko, A.V., Sharko, F.S., \& Velichkovsky, B.M. (2017). Exploring terra incognita of cognitive science: Lateralization of gene expression at the frontal pole of the human brain. Psychology in Russia: State of the Art, 10(3), 231-247. doi: 10.11621/pir.2017.0316

Hrvoj-Mihic, B., Bienvenu, T., Stefanacci, L., Muotri, A.R., \& Semendeferi, K. (2013). Evolution, development, and plasticity of the human brain: From molecules to bones. Frontiers in $\mathrm{Hu}$ man Neuroscience, 7, 707. doi: 10.3389/fnhum.2013.00707

James, W. (1890). The principles of psychology, Vols 1 and 2. New York: Henry Holt and Company. doi: 10.1037/10538-000

Kerestes, R., Chase, H.W., Phillips, M.L., Ladouceur, C.D., \& Eickhoff, S.B. (2017). Multimodal evaluation of the amygdala's functional connectivity. NeuroImage, 148, 219-229. doi: 10.1016/j.neuroimage.2016.12.023

Markett, S., Reuter, M., Heeren, B., Lachmann, B., Weber, B., \& Montag, C. (2017). Working memory capacity and the functional connectome - insights from resting-state fMRI and voxelwise centrality mapping. Brain Imaging and Behavior. doi: 10.1007/s11682-017-9688-9

Nedoluzhko, A.V., Efimova, O.I., Kildyushov, E.M., Dolina, I.A., Sokolov, A.S., Ushakov, V.L., Khaitovich, P.E. ... Velichkovsky, B.M. (in preparation). Sequencing frontopolar cortex: New insights into the molecular base of human cerebral asymmetry.

Panksepp, J. (1998). Affective neuroscience: The foundations of human and animal emotions. New York: Oxford University Press.

Piaget, J. (1972). Psychology and epistemology: Towards a theory of knowledge. Harmondsworth, U.K.: Penguin.

Ushakov, V.L. Sharaev, M.G., Kartashov, S.I., Zavyalova, V.V., Verkhlyutov, V.M., \& Velichkovsky, B.M. (2016). Dynamic Causal Modeling of hippocampal links within the human default mode network: Lateralization and computational stability of effective connections. Frontiers in Human Neuroscience, 10, 528, e64466. doi: 10.3389/fnhum.2016.00528

Velichkovsky, B.M. (1999). From levels of processing to stratification of cognition: Converging evidence from three domains of research. In B.H. Challis \& B.M. Velichkovsky (Eds). Stratification in cognition and consciousness (pp. 203-235). Amsterdam/Philadelphia: John Benjamins. doi: 10.1075/aicr.15.13vel

Velichkovsky, B.M. (2002). Heterarchy of cognition: The depths and the highs of a framework for memory research. Memory, 10(5/6), 405-419. doi: 10.1080/09658210244000234

Velichkovsky, B.M., Krotkova, O.A., Sharaev, M.G., \& Ushakov, V.L. (2017). In search of the "I": Neuropsychology of lateralized thinking meets Dynamic Causal Modeling. Psychology in Russia: State of the Art, 10(3), 7-27. doi: 10.11621/pir.2017.0301 